\title{
Description du fardeau de la dépression sur la santé de la population au Canada : utilisation de l'espérance de vie ajustée en fonction de la santé
}

\author{
C. Steensma, M. Sc. (1, 2); L. Loukine, M. Sc. (1); H. Orpana, Ph. D. (1, 3); L. McRae, B. Sc. (1); J. Vachon, M. Sc. (1);
} F. Mo, Ph. D. (1); M. Boileau-Falardeau, M. Sc. (1, 2); C. Reid, M.A. (1); B. C. Choi, Ph. D. (1, 4, 5)

Cet article a fait l'objet d'une évaluation par les pairs.

Diffuser cet article sur Twitter

\section{Résumé}

Introduction : Peu d'études ont évalué, dans l'ensemble d'une population, les conséquences de la dépression en matière de pertes dues à la mortalité prématurée d'une part et à la qualité de vie liée à la santé (QVLS) d'autre part. L'espérance de vie ajustée en fonction de la santé (EVAS) est une mesure synthétique de la santé de la population qui combine la morbidité et la mortalité en une seule statistique succincte décrivant l'état de santé d'une population à un moment donné.

Méthodologie : Nous avons estimé la QVLS de la population canadienne adulte en fonction de la présence ou non de dépression. Nous avons effectué un suivi de la mortalité, de 1994 à 2009, des participants à l'Enquête nationale sur la santé de la population (ENSP) (n = 12373) de 20 ans et plus, là aussi en fonction de la présence ou non de dépression. La dépression a été définie comme l'état d'une personne susceptible d'avoir connu au cours de l'année précédente un épisode dépressif majeur au sens du formulaire abrégé de l'Entrevue composite diagnostique internationale. L'espérance de vie a été estimée en créant des tables abrégées de mortalité selon le sexe et l'état dépressif à l'aide des risques relatifs de mortalité de l'ENSP et des données sur la mortalité du Système canadien de surveillance des maladies chroniques (2007 à 2009). L’Enquête sur la santé dans les collectivités canadiennes (2009-2010) a fourni des estimations de la prévalence de la dépression, et l'indice de l'état de santé Health Utilities Index a permis de mesurer la QVLS. L'EVAS de la population adulte a été mesurée en fonction de la présence ou non de dépression et en fonction du sexe à l'aide des estimations combinées de la mortalité, de la prévalence de la dépression et de la QVLS.

Résultats : Chez les femmes ayant connu récemment un épisode de dépression majeure, l'EVAS à 20 ans était de 42,0 ans (IC à $95 \%$ : 40,2 à 43,8), contre 57,0 ans (IC à $95 \%$ : 56,8 à 57,2 ) chez les femmes n'ayant pas vécu récemment d'épisode de dépression majeure. Au sein de la population masculine canadienne, l'EVAS à 20 ans était de 39,0 ans (IC à $95 \%$ : 36,5 à $41,5)$ chez ceux qui avaient connu récemment un épisode de dépression majeure, contre 53,8 ans (IC à $95 \%$ : 53,6 à 54,0) chez ceux n'ayant pas connu récemment d'épisode de dépression majeure. La différence de 15 ans dans l'EVAS des femmes avec et sans épisode récent de dépression majeure peut se décomposer en 12,3 ans attribuables à l'écart de la QVLS et 2,7 ans à l'écart observé en matière de mortalité. Les 14,8 années de moins d'EVAS chez les hommes ayant souffert de dépression correspondent à un écart de la QVLS de 13 ans et à un écart de mortalité de 1,8 an.

Conclusion : La population canadienne adulte atteinte de dépression au Canada avait une espérance de vie en santé considérablement plus faible que celle ne souffrant pas de dépression, chez les hommes comme chez les femmes. Si la majeure partie de cet écart s'explique par des niveaux moins élevés de la QVLS, la mortalité prématurée joue également un rôle.
Points saillants

- Au Canada, les hommes et les femmes qui vivent une dépression passent une proportion substantiellement plus importante de leur vie en mauvaise santé que la population non atteinte de dépression.

- L'écart observé dans l'espérance de vie en santé entre les Canadiens atteints de dépression et les autres est principalement attribuable à des pertes en matière de qualité de vie.

- L'état émotionnel, la capacité cognitive et la douleur sont les attributs clés associés à la diminution de la qualité de vie des Canadiens ayant vécu un épisode récent de dépression majeure.

- Le fardeau de la dépression sur l'espérance de vie en santé à l'échelle de la population canadienne semble plus important que celui associé à d'autres maladies chroniques comme le diabète, l'hypertension ou l'obésité, si l'on se fie aux observations d'autres études effectuées auprès des ménages canadiens.

Mots-clés : espérance de vie, espérance de vie en santé, mortalité, qualité de vie liée à la santé, dépression

\section{Introduction}

La dépression contribue de manière significative au fardeau des maladies, au Canada comme dans le reste du monde ${ }^{1}$. On estime que plus de 298 millions de personnes

Rattachement des auteurs :

1. Direction générale de la promotion de la santé et de la prévention des maladies chroniques, Agence de la santé publique du Canada, Ottawa (Ontario), Canada

2. Département de médecine sociale et préventive, Université de Montréal, Montréal (Québec), Canada

3. École de psychologie, Université d'Ottawa, Ottawa (Ontario), Canada

4. École de santé publique Dalla Lana, Université de Toronto, Toronto (Ontario), Canada

5. Centre de recherche sur la prévention des blessures, Collège médical de l'Université de Shantou, Shantou (Guangdong), Chine

Correspondance : Bernard Choi, Direction générale de la promotion de la santé et de la prévention des maladies chroniques, Agence de la santé publique du Canada, 785, avenue Carling, Ottawa (Ontario) K1A 0K9; tél. : 613-797-3821; téléc. : 613-941-2057; courriel : Bernard.Choi@phac-aspc.gc.ca 
dans le monde vivent avec une dépression ${ }^{2}$. En 2012, environ 3,2 millions de Canadiens de plus de 15 ans (11,3\%) ont rapporté avoir eu au cours de leur vie des symptômes s'apparentant à ceux d'un épisode de dépression majeure, la prévalence d'un tel épisode au cours des 12 mois précédents s'élevant à 4,7\% dans l'ensemble de la population ${ }^{3}$. C'est chez les femmes et les jeunes de 15 à 24 ans que la prévalence d'un épisode de dépression majeure de 12 mois était la plus élevée ${ }^{3}$.

La dépression a un impact sur la qualité de vie liée à la santé (QVLS), les capacités fonctionnelles, la mortalité due à une blessure intentionnelle et l'utilisation des soins de santét-6. Il a également été prouvé, outre ces effets négatifs directs, que la dépression augmente le risque de coronaropathie ${ }^{7}$, d'accident vasculaire cérébral ${ }^{8}$ et de cancer', ainsi qu'un déclin du fonctionnement physique $^{10}$. Les mécanismes potentiels de la relation entre la dépression et la maladie physique relèvent notamment du dérèglement des systèmes immunitaire et endocrinien et des processus inflammatoires ${ }^{11}$. La dépression a aussi été associée à un risque accru de mortalité, que ce soit dans l'ensemble de la population ou chez les patients atteints de maladies chroniques comme les coronaropathies, le cancer, le diabète ou les $\mathrm{AVC}^{11}$. Des effets réciproques existent probablement entre la dépression et la maladie, la dépression constituant à la fois un facteur de risque et une conséquence de la maladie ${ }^{11}$.

Des mesures globales de l'état de santé de la population effectuées à l'aide de la méthodologie de mesure du fardeau mondial de la maladie (Global Burden of Disease) $)^{1,12-15}$ ont accordé à la dépression une place très élevée sur la liste des problèmes de santé contribuant au fardeau de la maladie à l'échelle nationale et mondiale, particulièrement en matière de pertes résultant d'une incapacité. Vivre un épisode de dépression majeure arrive au deuxième rang des causes à des années vécues avec une incapacité, que ce soit à l'échelle mondiale ${ }^{1}$ ou aux États-Unis ${ }^{12}$, au Royaume-Uni ${ }^{13}$, en Chine ${ }^{14}$ et au Canada ${ }^{15}$.

On en sait moins sur l'association entre la dépression et l'espérance de vie ainsi que l'espérance de vie en santé (ou exempte de maladie). Comprendre l'espérance de vie et l'espérance de vie en santé chez les personnes atteintes de dépression contribuera à mieux caractériser le fardeau de la maladie. Une revue systématique et une métaanalyse ${ }^{16}$ ont permis de conclure que le taux de mortalité des personnes vivant avec un trouble de l'humeur est deux fois plus élevé que celui des personnes sans trouble de ce type, et que le nombre potentiel d'années perdues en raison de troubles mentaux varie entre 1,4 et 32 , la médiane étant de 10,1 ans. Jia et collab. ${ }^{17}$ ont rapporté que, aux États-Unis, à l'âge de 18 ans, les adultes souffrant de dépression subissent une perte d'espérance de vie pondérée par la qualité de l'existence (EVPQE) de 28,9 ans par rapport aux personnes ne souffrant pas de dépression. Les résultats des rares études disponibles sur l'espérance de vie en santé ou exempte de maladie et la dépression ne sont pas concluants ${ }^{18-20}$. Un certain nombre d'entre elles ne portent que sur les adultes âgés et ne peuvent donc s'appliquer à l'ensemble de la population. Ces études se fondent, de plus, sur des mesures de la santé fonctionnelle qui se limitent aux activités quotidiennes, sans tenir compte d'attributs comme la douleur, l'état émotionnel ou la capacité cognitive.

L'objectif de notre étude était d'estimer l'espérance de vie du moment (EV) et l'espérance de vie ajustée en fonction de la santé (EVAS) des adultes canadiens (âgés de 20 ans et plus) en fonction de présence ou non de dépression. Il convient de noter qu'en ce qui concerne la dimension temporelle, l'approche adoptée pour estimer l'EV et l'EVAS dans cet article consiste en une mesure synthétique de l'état de santé de la population pour une période donnée. L'espérance de vie à un moment donné permet d'estimer l'espérance de vie hypothétique d'une personne si celle-ci se conformait au taux de mortalité en fonction de l'âge et du sexe la concernant à ce moment-là, ce qu'on ne doit pas confondre avec l'espérance de vie projetée en fonction de l'espérance de vie modélisée ou d'une cohorte, qui prend pour référence le taux de mortalité réel de cette cohorte. De la même façon, l'EVAS du moment est une estimation hypothétique représentant l'espérance de vie en santé d'une personne si elle se conformait au taux de mortalité en fonction de l'âge et du sexe ainsi qu'au niveau de QVLS en fonction de l'âge et du sexe à ce moment-là.

Ces estimations sont utiles pour mieux comprendre l'impact d'une maladie sur la santé d'une population. Elles sont aussi utiles pour orienter les politiques et les programmes et pour prendre des décisions concernant le fardeau relatif de certains problèmes de santé. En raison des formes variées que peut prendre la dépression, et les cas chroniques comme épisodiques étant pris en compte dans la population à l'étude, nos estimations ne devraient pas servir à prédire l'évolution de l'état de santé d'un individu.

\section{Méthodologie}

\section{Sources des données}

Pour estimer l'EVAS, plusieurs types de données sont nécessaires : les taux de mortalité toutes causes confondues en fonction de la présence ou non de dépression, les estimations de prévalence de la dépression et les estimations de la QVLS en fonction de la présence ou non de dépression. Les taux de mortalité toutes causes confondues de la population canadienne adulte en fonction de la présence ou non de dépression ont été estimés selon une méthodologie qui répartit les taux de mortalité toutes causes confondues de l'ensemble de la population en taux de mortalité par catégories de maladies, à l'aide des rapports de risques relatifs de mortalité et de la prévalence de ces catégories. Cette méthodologie est décrite en détail ailleurs ${ }^{21}$.

Nous avons utilisé les données des trois sources suivantes :

- L'Enquête nationale sur la santé de la population (ENSP), pour l'estimation des risques de mortalité selon la présence ou non de dépression;

- L’Enquête sur la santé dans les collectivités canadiennes (ESCC), pour l'estimation de la prévalence de la dépression et la QVLS selon la présence ou non de dépression;

- Le Système canadien de surveillance des maladies chroniques (SCSMC), pour obtenir les taux de mortalité réels de la population canadienne toutes causes confondues, selon l'âge et le sexe, lesquels ont ensuite été répartis selon la présence ou non de dépression, en fonction du rapport de risques de mortalité estimé, à l'aide de l'ENSP, et de la prévalence de la dépression estimée par l'ESCC.

L'ENSP est une enquête longitudinale qui a été menée par Statistique Canada auprès de 17276 Canadiens de tous âges vivant 
dans un ménage et qui a couvert les dix provinces. L'ENSP a procédé à un suivi bisannuel entre 1994-1995 et 2010-2011 consistant entre autres à confirmer les décès en utilisant la Base canadienne de données sur la mortalité22. Ces données ont été utilisées pour procéder à une estimation des risques relatifs de mortalité associés à la dépression, une mesure nécessaire pour l'estimation des taux de mortalité toutes causes confondues des personnes atteintes de dépression et de celles non atteintes de dépression. La population ayant fait l'objet de notre étude était composée à la base de 12373 personnes âgées de 20 ans et plus.

L'ESCC est une enquête transversale effectuée chaque année par Statistique Canada auprès d'un échantillon composé d'environ 65000 Canadiens âgés de 12 ans et plus et vivant dans un ménage, à l'échelle de l'ensemble des provinces et territoires ${ }^{23}$. Pour notre étude, nous avons utilisé un échantillonnage de l'ESCC de deux ans (2009-2010) de 103815 participants âgés de 20 ans et plus pour estimer la prévalence de la dépression et pour associer la présence et l'absence de dépression aux taux de mortalité toutes causes confondues (voir la section "Analyse » pour plus de détails). Nous avons également obtenu de l'ESCC les scores de l'indice de l'état de santé (IES) selon la présence ou non de dépression. Nous avons mesuré la prévalence de la dépression et les scores de l'IES à partir des données de l'ESCC parce que celles-ci étaient plus récentes et que la taille plus importante de l'échantillon nous permettait de procéder à une estimation plus précise autant de la dépression que des scores de l'IES.

Nous avons utilisé également pour notre étude les données du SCSMC sur la mortalité toutes causes confondues pour 20072009. Le SCSMC recueille, à l'échelle de l'ensemble des provinces et territoires, des données administratives, dont les effectifs, par sexe et par tranches d'âge de cinq ans, de tous les décès et de tous les résidents utilisant un système de soins de santé public indépendamment de leur âge. Les données concernant la mortalité et la taille de la population proviennent des bases de données de l'assurance-maladie, qui couvre environ $97 \%$ de la population canadienne. Les données sont recueillies pour l'ensemble des provinces et territoires du Canada et synthétisées par l'Agence de la santé publique du Canada ${ }^{24}$.

\section{Mesures}

La dépression est mesurée, dans l'ESCC comme dans l'ENSP, à l'aide du formulaire abrégé de l'Entrevue composite diagnostique internationale (CIDI), un instrument diagnostique structuré, conçu pour produire des diagnostics conformes aux définitions et critères de la Classification internationale des maladies (CIM-10) et du Manuel diagnostique et statistique des troubles mentaux (DSM-IV). Chaque entrevue est menée par des enquêteurs qualifiés. La version abrégée de l'entrevue est fondée sur un sous-ensemble de questions de la CIDI capables de reproduire de manière fiable les estimations de prévalence $^{25}$. Elle fournit des scores qui permettent de prédire la probabilité de dépression. Dans le cadre de cette étude, les répondants dont la probabilité prédite atteignait 0,9 ou plus ont été considérés comme ayant connu un épisode de dépression majeure au cours de l'année précédente. Notons que le formulaire abrégé du CIDI est un module à contenu facultatif de l'ESCC 2009-2010, et toutes les provinces et tous les territoires n'ont pas fait le choix de l'incorporer. Les estimations de la dépression correspondent donc à une couverture provinciale et territoriale partielle, de cinq provinces (Île-du-Prince-Édouard, Québec, Saskatchewan, Alberta et ColombieBritannique) et deux territoires (Nunavut et Territoires du Nord-Ouest).

La QVLS est mesurée dans l'ESCC à l'aide de l'indice de l'état de santé Health Utilities Index Mark 3 (IES3), une mesure fondée sur les préférences sociétales à partir des réponses aux questions sur la santé fonctionnelle globale d'une personne portant sur huit attributs : la vision, l'ouie, l'élocution, la mobilité, la dextérité, les émotions, la cognition et la douleur ${ }^{26}$. Chaque attribut fonctionnel se voit accorder un score se situant entre 0,0 (plus bas niveau de fonctionnement) et 1,0 (pleine capacité fonctionnelle). Les huit attributs sont combinés pour obtenir un résultat d'ensemble dont les valeurs se situent entre 1,00 (parfaite santé) et - 0,36 (le pire état de santé possible, sachant que du point de vue des préférences, certains états de santé sont considérés comme pires que la mort et se voient donc attribuer des scores négatifs, 0,00 correspondant au décès). Des variations de 0,03 ou plus dans le score d'ensemble de l'IES et de 0,05 ou plus dans le score d'un attribut particulier sont considérées comme significatives du point de vue clinique ${ }^{27}$.

L'IES a été largement utilisé, et sa validité et sa fiabilité pour de nombreuses applications ont été prouvées ${ }^{27,28}$. Une étude visant à évaluer les résultats concernant la sensibilité à la dépression de plusieurs indices à attributs multiples a permis de déterminer que l'IES était capable de distinguer de façon efficace les niveaux de gravité dans les deux instruments de dépistage de la dépression évalués ${ }^{29}$. Le score à l'IES a été utilisé comme mesure de la morbidité dans l'estimation de l'EVAS. La dépression a généralement une incidence défavorable sur l'état émotionnel, en particulier un affect négatif soutenu et des difficultés à connaître un affect positif ${ }^{30}$. Pour déterminer si les différences de la QVLS étaient exclusivement dues aux changements de l'attribut associé aux émotions de l'IES, nous avons comparé les scores de chacun des attributs de l'IES (tous âges confondus) en fonction de la présence ou non de dépression.

\section{Analyse statistique}

Les risques relatifs de mortalité selon la présence ou non de dépression ont été estimés chez les femmes et les hommes à l'aide des rapports de risques (RR). Ces RR ont été déterminés à l'aide des données de l'ENSP, en procédant à un ajustement des modèles proportionnels à temps discret selon le sexe à une fonction bilogarithmique complémentaire. Nous avons défini les personnes atteintes de dépression comme celles qui présentaient une probabilité élevée $(0,9)$ d'avoir connu un épisode dépressif au cours des 12 mois précédant la collecte des données d'au moins un cycle de l'ENSP, conformément à la méthode décrite par Simpson et collab. ${ }^{31}$. Le premier épisode a permis d'associer une date au cas. Ensuite, un suivi de la mortalité des répondants a été effectué tous les deux ans et une variable pour chaque cycle a été incluse dans le modèle (cycle 1 à cycle 9) comme mesure de l'intervalle temporel. Les modèles selon le sexe ont été ajustés en fonction de l'âge. Comme les RR servaient à estimer uniquement les taux de mortalité toutes causes confondues associés à la dépression, nous n'avons pas procédé à l'ajustement des modèles à d'autres comorbidités, aux situations socioéconomiques ou à tout autre déterminant de la santé. Du fait de la conception complexe de l'échantillonnage de cette 
étude, nous avons utilisé la méthode bootstrap pour calculer la variance et produire des intervalles de confiance (IC) à $95 \%$ pour le $\mathrm{RR}^{22}$. Les prévalences de dépression selon l'âge et le sexe, nécessaires pour décomposer les taux de mortalité totaux en fonction de la présence ou non de dépression, ont été estimées à l'aide des données de l'ESCC 2009-2010. Les estimations des IES moyens selon l'âge, le sexe et la présence ou non de dépression ont également été calculées. La méthode bootstrap a permis de générer un IC à $95 \%{ }^{23}$. Dans le cadre de notre étude, les taux de mortalité totaux selon le sexe et par tranches de cinq ans ont été estimés à partir des données du SCSMC pour la période 2007-2009. Les taux de mortalité selon l'âge et le sexe des personnes atteintes de dépression et des autres ont été obtenus en décomposant les taux de mortalité de l'ensemble de la population à l'aide de la méthodologie décrite dans une étude précédente ${ }^{21}$.

Nous avons utilisé la méthode de Chiang ${ }^{32}$ pour produire des tables de mortalité selon le sexe et la présence ou non de dépression pour la période (2007-2009) à l'aide de 14 groupes d'âge standard (20 à 24,25 à $29, \ldots, 80$ à 84,85 ans et plus). La fonction de Gompertz a servi à fournir un estimé précis de l'EV pour le dernier intervalle d'âge de 85 ans et plus, qui était ouvert, afin de confirmer la table de mortalité, comme le décrit Hsieh ${ }^{33}$. Une méthode de Sullivan modifiée ${ }^{34}$ a été utilisée pour estimer l'EVAS. Les " années de vie vécues " ont été ajustées en utilisant l'IES, conformément à cette méthode :

$$
V_{x}^{\prime}=V_{x} * I E S_{x}
$$

où $V_{x}^{\prime}$ correspond aux années de vie vécues ajustées à un intervalle d'âge $x$, où $V_{x}$ correspond aux années de vie vécues pour l'intervalle d'âge $x$ et où IES ${ }_{x}$ est l'indice Index Mark 3 s'appliquant aux personnes dans l'intervalle d'âge $x$.

La variance de l'EV et de la QVLS a été calculée à l'aide de la méthode bootstrap. Les enquêtes de Statistique Canada fournissent 500 poids bootstrap pour l'estimation de la variance afin de tenir compte des plans d'enquêtes complexes ${ }^{22,23}$. À l'aide de ces poids, 500 ensembles d'estimation du RR de l'ENSP et 500 ensembles de prévalence de la dépression et d'estimation de l'IES de l'ESCC ont été générés, et l'ensemble des combinaisons spécifiques de ces estimations a permis d'obtenir des taux de mortalité qui ont servi à l'élaboration de 250000 tables de mortalité selon le sexe et la présence ou non de dépression. Cela a permis d'estimer la variance de l'EV et de l'EVAS et de générer des tests $\mathrm{Z}$ visant à déterminer l'importance statistique des différences dans l'EV et l'EVAS. Un IC à $95 \%$ a été créé à partir de l'hypothèse de normalité. En raison de la nature de la population étudiée (20 ans et plus), l'EV et l'EVAS ont été estimées à 20 ans et non à la naissance.

La méthode de décomposition ou de répartition d'Arriaga ${ }^{35}$ (adaptation de la méthode de Sullivan ${ }^{34}$ ) a été appliquée pour quantifier quelle proportion des différences dans l'EVAS selon la présence ou non de dépression est attribuable à une mortalité prématurée et quelle proportion est attribuable à une perte de QVLS (morbidité). Pour chaque groupe d'âge, les changements dans l'EVAS entre groupes de comparaison ont été répartis entre les composantes suivantes :

$\triangle E V A S=\triangle M O R B+\triangle M O R T=\frac{V_{x 1}+V_{x 2}}{2} \Delta I E S+\frac{I E S_{1}+I E S_{2}}{2} \Delta V_{x}$

où $\triangle M O R B$ correspond à l'estimation du changement dû à la QVLS, $\triangle M O R T$, à l'estimation du changement dû à la mortalité, où $V_{x 1}$ et $I E S_{x 1}$ font respectivement référence au nombre d'années vécues et au score de l'IES des personnes atteintes de dépression dans un intervalle d'âge $x$, et $V_{x 2}$ et $H U I_{x 2}$ font respectivement référence au nombre d'années vécues et au score de l'IES des personnes qui ne sont pas atteintes de dépression dans un intervalle d'âge $x$. (Plus de détails au sujet de cette méthodologie appliquée à l'estimation de l'espérance de vie en santé sont disponibles ailleurs ${ }^{21,36}$.)

\section{Résultats}

Le tableau 1 présente les caractéristiques des participants à l'ENSP et à l'ESCC, les deux enquêtes nationales ayant servi à cette étude. La prévalence de l'épisode dépressif dans la population de notre étude (2009-2010) était de $5,5 \%(6,7 \%$ chez les femmes et 4,2\% chez les hommes, résultats non présentés).

\section{TABLEAU 1}

Caractéristiques des participants à l'enquête, ENSP 1994-1995 et ESCC 2009-2010

\begin{tabular}{|c|c|c|}
\hline Caractéristiques & ENSP 1994-1995 & ESCC 2009-2010 \\
\hline Âge (ans) (moyenne et amplitude) & 45 (20 à 100) & 47,5 (20 à 102) \\
\hline \multicolumn{3}{|l|}{ Sexe ${ }^{a} \%$ et IC à $\left.95 \%\right)$} \\
\hline Hommes & $48,6(48,3$ à 48,9$)$ & $49,1(49,1$ à 49,1$)$ \\
\hline Femmes & $51,4(51,1$ à 51,7$)$ & $50,9(50,9$ à 50,9$)$ \\
\hline \multicolumn{3}{|l|}{ Situation de famille (\% et IC à $95 \%$ ) } \\
\hline Mariés/Conjoints de fait & $68,3(67,3$ à 69,3$)$ & $65,7(65,1$ à 66,3$)$ \\
\hline Célibataires/Veufs/Divorcés/Séparés & $31,7(30,7$ à 32,7$)$ & $34,3(33,7$ à 34,9) \\
\hline \multicolumn{3}{|l|}{ Plus haut niveau de scolaritéc (\% et IC à $95 \%$ ) } \\
\hline Sans diplôme d'études secondaires & $25,7(24,5$ à 26,8$)$ & $14,4(14,1$ à 14,8$)$ \\
\hline Diplôme d'études secondaires & $40,7(39,5$ à 41,9) & $23,7(23,3$ à 24,2) \\
\hline Diplôme d'études postsecondaires & 33,6 (32,5 à 34,8) & $61,8(61,3$ à 62,4$)$ \\
\hline \multicolumn{3}{|l|}{ Dépressiond (\% et IC à $95 \%)$} \\
\hline Oui & S.o. & 5,5 (5,2 à 5,9) \\
\hline Non & S.o. & 94,5 (94,1 à 94,8) \\
\hline $\begin{array}{l}\text { Dépression d au cours d'au moins un cycle } \\
\text { (1994 à 2008) (\% et IC à } 95 \%)\end{array}$ & & - \\
\hline Oui & $4,7(4,4$ à 4,9) & s.o. \\
\hline Non & $95,3(95,0$ à 95,6) & s.o. \\
\hline
\end{tabular}

Abréviations : ENSP, Enquête nationale sur la santé de la population; ESCC, Enquête sur la santé dans les collectivités canadiennes; FA-CIDI, Formulaire abrégé de l'Entrevue composite diagnostique internationale; s.o., sans objet.

${ }^{a} \mathbf{n}=12373$ pour l'ENSP et $\mathbf{n}=103815$ pour l'ESCC.

${ }^{\mathrm{b}} \mathrm{n}=12371$ pour l'ENSP et $\mathrm{n}=103636$ pour l'ESCC.

${ }^{c} n=12347$ pour l'ENSP et $n=101783$ pour l'ESCC.

d À partir des réponses au formulaire abrégé de la CIDI, indiquant un trouble dépressif majeur au cours de l'année précédente. $\mathrm{n}=3501$ pour l'ENSP et $\mathrm{n}=48355$ pour l'ESCC. 
Selon l'analyse des données de l'ENSP, 2154 personnes sont décédées au cours de la période de suivi de 6 ans. Le risque de mortalité était significativement plus élevé chez celles qui avaient connu un épisode de dépression majeure (RR ajusté selon l'âge $=1,43$; IC à $95 \%: 1,22$ à 1,68). Un risque important était toujours observable avec limitation des analyses aux femmes (RR ajusté selon l'âge $=1,55$; IC à $95 \%$ : 1,28 à 1,87), alors que le risque chez les hommes seulement n'était pas significatif (RR ajusté selon l'âge $=1,28$; IC à $95 \%$ : 0,98 à 1,68) (données non présentées).

Les valeurs non ajustées de la QVLS (mesurées par les scores à l'IES) variaient selon l'âge, le sexe et la présence ou non de dépression (tableau 2). Les scores de l'IES étaient considérablement plus faibles chez les hommes et les femmes de tous les groupes d'âge ayant connu un épisode de dépression majeure au cours des 12 mois précédents, comparativement à ceux des personnes n'ayant pas vécu d'épisode de ce type. Si l'on applique les définitions des catégories d'incapacité fondées sur les scores globaux de l'IES déterminées par Feng et collab. ${ }^{37}$, les hommes et les femmes atteints de dépression ont connu, en moyenne, une incapacité modérée (IES < 0,89) dans tous les groupes d'âge, alors que, chez les hommes et les femmes non atteints de dépression, seuls les plus âgés (55 ans et plus) appartenaient à cette catégorie. De même, les hommes souffrant de dépression ont connu une incapacité grave (IES < 0,70) en moyenne à l'âge de 40 ans, alors que chez les femmes, une incapacité de ce type est apparue vers l'âge de 45 ans, et alors que la moyenne des scores à l'IES des hommes et des femmes sans dépression n'est jamais passée sous ce seuil, quel que soit l'âge, au sein de notre étude.

Une évaluation de chacun des huit attributs selon le sexe (tous âges confondus) montre que la dépression était associée à un score plus faible cliniquement significatif (c.-à-d. une différence de 0,05 ou plus) pour les attributs des émotions, de la douleur et de la cognition (tableau 2).

TABLEAU 2

État de la qualité de vie liée à la santé selon le sexe, l'âge, les attributs de l'IES et la présence ou non de dépressiona, Canada, 2009-2010

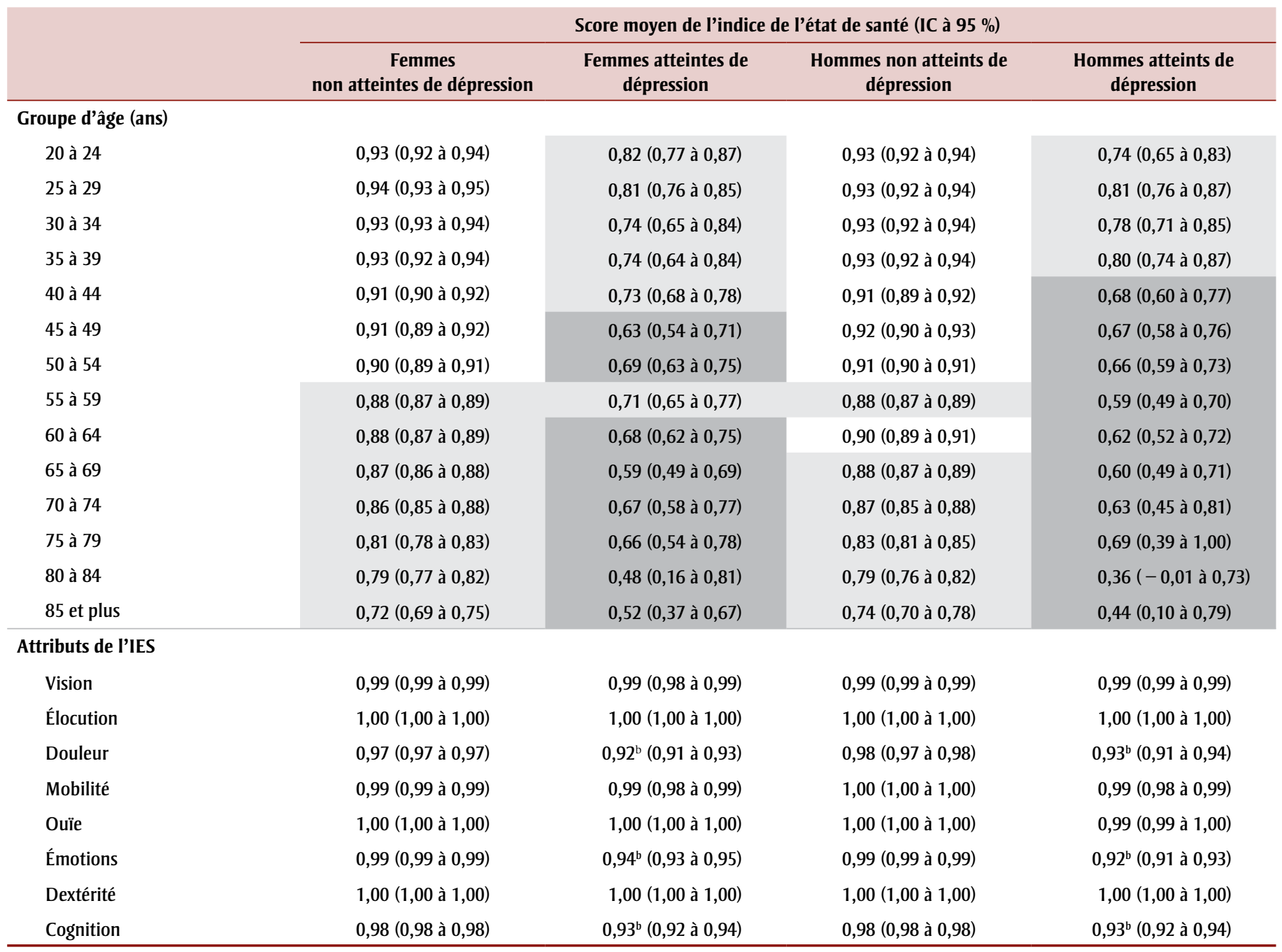

Abréviations : FA-CIDI, Formulaire abrégé de l'Entrevue composite diagnostique internationale; IC, intervalle de confiance; IES, Indice de l'état de santé.

Remarque : l'ombrage gris pâle indique une incapacité modérée (score global de l'IES de 0,70 à 0,88); l'ombrage foncé indique une incapacité grave (score global de l'IES inférieur à 0,70 ); l'absence d'ombrage indique l'absence d'incapacité (score global de l'IES de 1,00) ou une incapacité légère (score global de l'IES de 0,89 à 0,99).

a À partir des réponses au formulaire abrégé de la CIDI indiquant un trouble dépressif majeur au cours de l'année précédente.

${ }^{\text {b }}$ Différence cliniquement significative dans le score de l'IES concernant cet attribut entre ceux qui ont souffert de dépression et ceux qui n'en ont pas souffert. 
L'EV et l'EVAS des femmes atteintes de dépression étaient plus faibles que celles des femmes qui n'en étaient pas atteintes. L'EV des hommes atteints de dépression n'était significativement pas plus faible, alors l'EVAS l'était (tableau 3). À 20 ans, l'EV des femmes atteintes de dépression était de 4,1 ans (IC à $95 \%: 1,1$ à 7,1) plus courte et l'EVAS était de 15,0 ans (IC à $95 \%: 13,2$ à 16,8 ) plus faible, comparativement aux femmes qui n'en étaient pas atteintes. Chez les hommes, au même âge, l'écart de l'EV entre ceux atteints de dépression et ceux qui n'en étaient pas atteints correspondait à 2,7 ans (IC à $95 \%: 0,0$ à 5,4), alors que l'EVAS était de 14,8 ans (IC à $95 \%$ : 12,3 à 17,4) plus faible. L'EV et l'EVAS des hommes et des femmes atteints de dépression à 65 ans étaient plus faibles que celles des personnes qui n'en étaient pas atteintes. À cet âge, les femmes souffrant de dépression avaient une EV de 3,2 ans (IC à $95 \%: 1,8$ à 4,6 ) plus courte et une EVAS de 6,7 ans (IC à $95 \%: 5,3$ à 8,1) plus faible que celles qui n'étaient pas atteintes de dépression et les hommes dépressifs avaient une EV de 2,1 ans (IC à $95 \%: 0,1$ à 4,1 ) plus courte et une EVAS de 6,0 ans (IC à $95 \%$ : 3,8 à 8,2 ) plus faible que ceux qui n'étaient pas atteints de dépression.

Une évaluation de la contribution spécifique de la perte de la QVLS et de la mortalité prématurée aux différences observées dans l'EVAS à l'âge de 20 ans a indiqué qu'en ce qui concerne la différence de 15,0 ans de l'EVAS entre les femmes atteintes et non atteintes de dépression, 12,3 années étaient attribuables aux pertes de QVLS et les 2,7 autres années aux pertes liées à la mortalité. L'EVAS de 14,8 ans de moins chez les hommes atteints de dépression équivalait à un écart de la QVLS de 13,0 ans et à une perte de 1,8 an liée à la mortalité (données non présentées).

Nous avons constaté d'importantes différences entre les adultes canadiens atteints et non atteints de dépression en matière de pourcentage de vie marqué par des problèmes de santé ([EV-EVAS]/EV; voir figure 1). Les hommes comme les femmes qui souffrent de dépression passent près de trois fois plus de temps de leur espérance de vie à 20 ans en mauvaise condition physique que la population qui ne souffre pas de dépression (31\% contre $12 \%$ chez les femmes et $32 \%$ contre $11 \%$ chez les hommes). Ces écarts importants existent dans tous les groupes d'âge. En se fondant sur l'EV et l'EVAS à 65 ans, la période vécue en
TABLEAU 3

Espérance de vie et EVAS à l'âge de 20-24 ans et de 65-69 ans selon le sexe et la présence ou non de dépressiona ${ }^{\mathrm{a}}$, Canada, 2009-2010

\begin{tabular}{lccccc} 
& $\begin{array}{c}\text { Groupe d'âge } \\
\text { (ans) }\end{array}$ & $\begin{array}{c}\text { Femmes non } \\
\text { atteintes de } \\
\text { dépression }\end{array}$ & $\begin{array}{c}\text { Femmes } \\
\text { atteintes de } \\
\text { dépression }\end{array}$ & $\begin{array}{c}\text { Hommes non } \\
\text { atteints de } \\
\text { dépression }\end{array}$ & $\begin{array}{c}\text { Hommes } \\
\text { atteints de } \\
\text { dépression }\end{array}$ \\
\hline $\begin{array}{l}\text { Espérance de } \\
\text { vie }\end{array}$ & 20 à 24 & $\begin{array}{c}64,9 \\
(64,8 \text { à } 65,0)\end{array}$ & $\begin{array}{c}60,8 \\
(59,0 \text { à } 62,6)\end{array}$ & $\begin{array}{c}60,4 \\
(60,3 \text { à } 60,5)\end{array}$ & $\begin{array}{c}57,7 \\
(55,0 \text { à } 60,4)\end{array}$ \\
\cline { 2 - 6 } & 65 à 69 & $\begin{array}{c}22,4 \\
(22,3 \text { à } 22,5)\end{array}$ & $\begin{array}{c}19,2 \\
(17,8 \text { à } 20,6)\end{array}$ & $\begin{array}{c}19,1 \\
(19,1 \text { à } 19,1)\end{array}$ & $\begin{array}{c}17,0 \\
(15,0 \text { à } 19,0)\end{array}$ \\
\hline $\begin{array}{l}\text { Espérance de } \\
\text { vie ajustée en } \\
\text { fonction de la } \\
\text { santé }\end{array}$ & 20 à 24 & 57,0 & 42,0 & 53,8 & 39,0 \\
\hline
\end{tabular}

Abréviations : EVAS, espérance de vie ajustée en fonction de la santé; FA-CIDI, Formulaire abrégé de l’Entrevue composite diagnostique internationale; IC, intervalle de confiance.

a À partir des réponses au formulaire abrégé de la CIDI indiquant un trouble dépressif majeur au cours de l'année précédente. mauvaise santé par les hommes et les femmes souffrant de dépression était deux fois plus longue que pour le reste de la population (40\% contre $19 \%$ chez les femmes et $43 \%$ contre $17 \%$ chez les hommes).

\section{Analyse}

Dans le cadre de cette étude, nous avons conclu que l'EV à 20 ans chez les femmes et l'EVAS à 20 ans chez les femmes et chez les hommes étaient beaucoup plus faibles chez les Canadiens ayant rapporté des symptômes s'apparentant à ceux d'un épisode de dépression majeure au cours des 12 mois précédents. Nous avons constaté ce fait dans tous les groupes d'âge, avec cependant des écarts dans la proportion de l'espérance de vie marquée par des problèmes de santé plus grands chez les hommes et les personnes plus jeunes souffrant

\section{FIGURE 1}

Pourcentage de la vie marqué par des problèmes de santéa, selon le sexe, l'âge et l'état dépressif b', Canada, 2009-2010

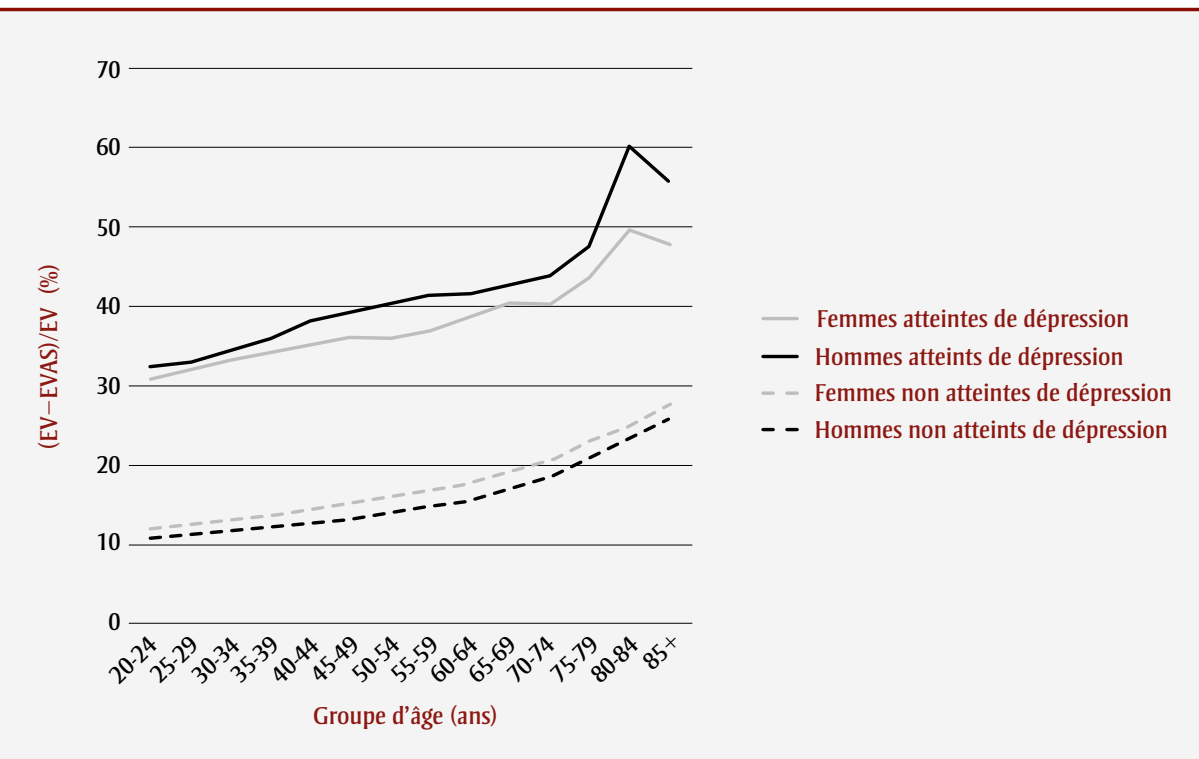

Abréviations : EVAS, espérance de vie ajustée en fonction de la santé; FA-CIDI, Formulaire abrégé de l’Entrevue composite diagnostique internationale.

a (EV-EVAS)/EV.

${ }^{\mathrm{b}}$ À partir des réponses au formulaire abrégé de la CIDI indiquant un trouble dépressif majeur au cours de l'année précédente. 
de dépression. Si les comparaisons directes avec d'autres problèmes de santé sont à interpréter avec prudence, nous avons constaté que les pertes de QVLS associées à la dépression dans la population canadienne adulte étaient plus importantes que celles observées au sein de la même population en association avec l'obésité de classe 2 et au-delà ${ }^{21}$, le diabète et $l^{\prime}$ hypertension ${ }^{38}$. De plus, alors que ces études démontrent une diminution plus importante de l'EVAS chez les femmes que chez les hommes, nous avons, pour notre part, constaté des pertes similaires chez les deux sexes.

Nos résultats concordent avec ceux de Jia et collab. ${ }^{17}$, qui ont rapporté une perte de l'espérance de vie pondérée par la qualité de l'existence (EVPQE) de 28,9 ans, à l'âge 18 ans, chez les adultes souffrant de dépression, ce qui est substantiellement plus important que la perte approximative de 15 ans de l'EVAS à l'âge de 20 ans chez les adultes souffrant de dépression que nous avons rapportée. Cela peut s'expliquer par des différences réelles entre l'expérience de la population canadienne et celles de la population américaine en matière de santé ainsi que par des différences méthodologiques entre nos études. De plus, les rapports de risque de mortalité associés à la dépression que nous avons observés $(\mathrm{RR}=1,43)$ sont en quelque sorte moins élevés que le risque relatif de mortalité de 2,08 des adultes atteints de dépression rapportés dans la méta-analyse de Walker et collab. ${ }^{16}$. Les études effectuées sur des populations adultes plus âgées indiquent une moins grande espérance de vie chez les participants atteints de dépression. Tant Chiao et collab. ${ }^{18}$ que Pérès et collab. ${ }^{20}$ rapportent une diminution de l'espérance de vie à 65 ans d'approximativement un an chez les participants souffrant de dépression, alors que Reynolds et collab. ${ }^{19}$ ont constaté que l'espérance de vie à 70 ans des personnes atteintes de dépression, sans autre maladie chronique, était réduite d'environ trois ans. Bien que ces études démontrent une diminution de l'espérance de vie chez les participants vivant avec la dépression, l'ampleur de ces diminutions est moindre que celle que nous avons constatée dans notre étude, à l'exception de celle des participants masculins de l'étude de Reynolds et collab. ${ }^{19}$. Les différences les plus importantes peuvent être dues au fait que notre mesure de la QVLS intégrait des attributs qui ne se retrouvaient pas dans la mesure de l'incapacité relative aux activités de la vie quotidienne utilisée dans ces trois études.

Nous avons constaté que, dans une grande proportion, une faible EVAS chez les participants atteints de dépression était attribuable à des niveaux inférieurs de QVLS. Une comparaison de chacun des attributs de l'IES (tableau 2) a démontré que, bien qu'il existe une différence cliniquement significative entre les hommes et les femmes atteints et non atteints de dépression en ce qui a trait aux émotions, on constate également des différences significatives en ce qui a trait à la douleur et à la cognition. Si l'association de la douleur à la dépression est bien documentée, leur relation est complexe et les liens de causalité ne sont pas entièrement compris : les données confirment aussi bien le modèle où la dépression entraîne des changements chez la personne qui augmentent sa vulnérabilité à la douleur que les scénarios où les symptômes de la douleur sont un facteur de risque de dépression future ${ }^{39}$. Une dysfonction cognitive a également été observée chez une grande proportion de patients atteints de dépression et il a été démontré que celle-ci apparaît tôt dans l'évolution de la dépression, peut-être même avant l'établissement du diagnostic ${ }^{40}$.

Outre les valeurs inférieures attendues de l'EVAS en association avec une plus faible QVLS, nous avons aussi constaté qu'une proportion considérable de cette diminution est attribuable à une mortalité prématurée. Alors que nos résultats démontrent de plus grandes pertes, en matière d'espérance de vie, chez les femmes, d'autres études portant sur le risque de mortalité et l'espérance de vie en fonction de la dépression indiquent que les hommes présentent un risque plus élevé de mortalité ou de perte d'espérance de vie ${ }^{11,17,19,20}$. La plupart de ces études portent toutefois sur des populations d'aînés ou d'adultes plus âgés, qui ont un profil de risque différent de celui de la population adulte dans son ensemble. Shah et collab. ${ }^{41}$ ont en effet procédé à une évaluation des différences de l'association de la dépression et de la mortalité, selon l'âge et le sexe, et des interactions dépression-âge-sexe significatives ont été observées : le risque de mortalité s'accroissait après 55 ans chez les hommes au fur et à mesure que l'âge avançait, alors que la tendance inverse était observée chez les femmes. D’autres études demeurent à effectuer auprès de populations plus jeunes afin de mieux comprendre ce phénomène.

\section{Forces et limites}

Notre étude a bénéficié de données détaillées pour l'estimation de l'EV et de l'EVAS chez les adultes de tous âges au Canada. Les données d'enquête qui ont été utilisées proviennent d'importants échantillonnages de la population canadienne : l'ENSP nous a permis de procéder à un suivi de l'expérience de la mortalité de plus de 17000 adultes canadiens pendant 16 ans, une période plus longue que toute autre étude visant à évaluer l'association de l'état dépressif et de l'espérance de vie ou de l'espérance de vie en santé.

Notre étude comporte cependant plusieurs limites. Lors de l'estimation du risque de mortalité, nous n'avons consigné que le premier épisode de dépression probable, à partir des réponses au formulaire abrégé de la CIDI, et nous avons négligé l'état dépressif lors du suivi subséquent. Cela a pu entraîner une classification erronée de sujets dont la situation vis-à-vis de la dépression a changé.

La définition de la dépression utilisée dans cette étude (probabilité prévue d'un épisode de dépression majeure de 0,9 ) est conforme aux recommandations du FA-CIDI et correspond à la déclaration de cinq à neuf symptômes évocateurs de la dépression, ou d'un ou deux symptômes dominants. Cette mesure a été mise au point aux États-Unis pour la National Comorbidity Survey. Une probabilité de 0,9 est un seuil élevé se traduisant par un plus grand nombre de faux négatifs que de faux positifs, conduisant à sous-estimer et non à surestimer le fardeau de la dépression au Canada ${ }^{42}$.

Comme le FA-CIDI est un élément facultatif de l'ESCC, il n’intègre pas les réponses des Canadiens de toutes les provinces et tous les territoires, ce qui était susceptible de limiter la représentativité de nos résultats. Nous avons donc évalué les conséquences de l'absence de renseignements à l'aide d'un cycle antérieur de l'ESCC (2000-2001) intégrant les résultats des épisodes de dépression majeure de la totalité des provinces et territoires. Ainsi, en retirant de l'enquête antérieure les mêmes données que celles qui manquaient dans le cycle 2009-2010, la prévalence des épisodes de dépression majeure selon l'âge et le sexe de ce cycle n'avait pas changé de manière appréciable, suggérant que la représentativité de la population ayant servi à notre étude n'a pas été affectée par l'absence de personnes de 
certaines provinces et certains territoires. Il convient également de mentionner que notre mesure de la dépression, à savoir un épisode récent de dépression majeure, ne capte pas de manière adéquate les pertes, en matière d'espérance de vie en santé, associées plus spécifiquement à la dépression chronique ou à long terme.

L'ESCC est un sondage effectué auprès des ménages, excluant d'autres populations comme celles qui vivent en résidence ou dans des établissements de soins de longue durée, ce qui fait que la prévalence de la dépression ne reflète pas celle de la population canadienne dans son intégralité. Les non-réponses peuvent aussi entraîner des différences au sein de l'ENSP et de l'ESCC : les personnes atteintes de dépression peuvent être moins enclines à répondre, ce qui pourrait donner lieu là aussi à une sousestimation de la prévalence de la dépression. Cela signifierait que nos estimations sont globalement conservatrices et que le véritable fardeau de la dépression peut être plus élevé que ce que nous avançons.

Notre étude visait à décrire l'association entre la dépression et l'espérance de vie en santé et ne cherchait pas à comprendre l'influence modificatrice que peuvent exercer la situation socioéconomique ou d'autres problèmes de santé. Toutefois, il serait inapproprié, en décrivant la mortalité et la morbidité des personnes atteintes de dépression, de les ajuster en fonction des troubles comorbides. L'influence sur l'espérance de vie en santé des problèmes de santé qui présentent une comorbidité avec la dépression n'est pas claire. Si Pérès et collab. ${ }^{20}$ ont relevé des différences significatives dans l'espérance de vie en santé entre les personnes atteintes et non atteintes de dépression ayant signalé trois problèmes de santé chroniques ou plus, Reynolds et collab. ${ }^{19}$ ont, pour leur part, constaté des différences significatives dans l'espérance de vie en santé lorsqu'ils ont comparé des personnes atteintes de dépression avec d'autres non atteintes de dépression et que les sujets des deux groupes ne présentaient pas de maladie chronique. Des études plus approfondies sont nécessaires pour déterminer les répercussions de ces facteurs de risque et d'autres effets modificateurs potentiellement positifs, comme la participation sociale, sur l'espérance de vie en santé ${ }^{18}$.

Enfin, l'approche visant à faire la synthèse de l'état de santé de la population dans cette étude traite de l'espérance de vie et de vie en santé d'une population à un moment donné, en fonction des estimations de la mortalité et de la QVLS par âge et par sexe. Ces estimations de l'espérance de vie et de l'EVAS à un moment donné ne devraient être interprétées que comme des mesures synthétiques de l'état de santé de la population et non comme l'espérance de vie et de vie en santé attribuable à un individu en particulier.

\section{Conclusion}

Cette étude montre qu'à l'échelle de la population canadienne, les femmes ayant récemment connu un épisode de dépression majeure ont une espérance de vie et une EVAS considérablement plus faibles à 20 ans que celles qui n'ont pas vécu un épisode de dépression de ce type, et que pour les hommes, l'EVAS à 20 ans est considérablement plus faible chez ceux qui ont récemment connu un épisode de dépression majeure. Les diminutions de l'EVAS attribuables à une QVLS plus faible sont très importantes et, si elles ne présentent pas la même envergure, les pertes attribuables à une mortalité accrue contribuent elles aussi à cette différence, particulièrement chez les femmes. Ces observations témoignent du lourd fardeau de la dépression au sein de la population canadienne.

\section{Remerciements}

Cette recherche n’a pas été financée par une subvention d'un organisme des secteurs public, privé ou sans but lucratif. Il n’y a eu aucun conflit d'intérêts.

Colin Steensma a participé à la conception de l'étude, rédigé la première ébauche de cet article et en a fait l'examen critique. Lidia Loukine a participé à la conception de l'étude, mené toutes les analyses statistiques, rédigé certaines parties du présent article, dont elle aussi fait l'examen critique. Heather Orpana a participé à la conception de l'étude, rédigé certaines parties du présent article, dont elle aussi fait l'examen critique. Louise McRae a participé à la conception de l'étude et procédé à l'examen critique de l'article. Julie Vachon a contribué à l'analyse des études et à l'interprétation des données et procédé à l'examen critique de l'article. Frank Mo, Michèle Boileau-Falardeau et Carrie Reid ont participé à l'interprétation des données et procédé à l'examen critique de l'article. Bernard Choi a participé à la conception de l'étude et procédé à l'examen critique de l'article.

L'accès aux données ayant servi à cette étude a été octroyé par le biais d'accords de partage conclus avec Statistique Canada et les provinces et territoires canadiens. D'autres chercheurs peuvent avoir accès aux données de Statistique Canada au moyen son Initiative de démocratisation des données (IDD).

\section{Références}

1. Ferrari AJ, Charlson FJ, Norman RE et collab. Burden of depressive disorders by country, sex, age, and year: findings from the Global Burden of Disease Study 2010. PLoS Med. 2013; 10(11):e1001547. doi: 10.1371/journal. pmed.1001547.

2. Ferrari AJ, Charlson FJ, Norman RE et collab. The epidemiological modelling of major depressive disorder: application for the Global Burden of Disease Study 2010. PloS One. 2013;8(7): e69637. doi: 10.1371/journal.pone .0069637 .

3. Pearson C, Janz T, Ali J. Troubles mentaux et troubles liés à l'utilisation de substances au Canada. Ottawa (Ont.) : Statistique Canada; 2013.

4. Ruo B, Rumsfeld JS, Hlatky MA, Liu $\mathrm{H}$, Browner WS, Whooley MA. Depressive symptoms and healthrelated quality of life: the Heart and Soul Study. JAMA. 2003;290(2): 215-221. doi: 10.1001/jama.290.2.215.

5. Rihmer Z. Suicide risk in mood disorders.CurrOpinPsychiatry.2007;20(1):1722. doi: $10.1097 /$ yco.0b013e3280106868.

6. Stephens T, Joubert N. Le fardeau économique des problèmes de santé mentale au Canada. Maladies chroniques au Canada. 2001; 22(1):19-25.

7. Brown AD, Barton DA, Lambert GW. Cardiovascular abnormalities in patients with major depressive disorder. CNS Drugs. 2009;23(7): 583-602. doi: 10 .2165/00023210-200923070-00004.

8. Pan A, Sun Q, Okereke OI, Rexrode $\mathrm{KM}, \mathrm{Hu}$ FB. Depression and risk of stroke morbidity and mortality: a meta-analysis and systematic review. JAMA. 2011;306(11):1241-1249. doi: 10.1001/jama.2011.1282. 
9. Currier MB, Nemeroff CB. Depression as a risk factor for cancer: from pathophysiological advances to treatment implications. Annu Rev Med. 2014; 65:203-221. doi: 10.1146/annurev-med -061212-171507.

10. Penninx BW, Guralnik JM, Ferrucci L, Simonsick EM, Deeg DJ, Wallace RB. Depressive symptoms and physical decline in community-dwelling older persons. JAMA. 1998;279(21):1720-6. doi: 10.1001/jama.279.21.1720.

11. Cuijpers P, Vogelzangs N, Twisk J, Kleiboer A, Li J, Penninx BW. Is excess mortality higher in depressed men than in depressed women? A metaanalytic comparison. J Affect Disord. 2014;161:47-54. doi: 10.1016/j.jad.2014 .03 .003 .

12. Murray CJ, Abraham J, Ali MK et collab. The state of US health, 1990-2010: burden of diseases, injuries, and risk factors. JAMA. 2013;310(6):591-606. doi: 10.1001/jama.2013.13805.

13. Murray CJ, Richards MA, Newton JN et collab. UK health performance: findings of the Global Burden of Disease Study 2010. Lancet. 2013; 381(9871):997-1020. doi: 10.1016/ s0140-6736(13)60355-4

14. Yang G, Wang Y, Zeng Y et collab. Rapid health transition in China, 1990 à 2010: findings from the Global Burden of Disease Study 2010. Lancet. 2013;381 (9882):1987-2015. doi: 10.1016 /s0140-6736(13)61097-1.

15. Ratnasingham S, Cairney J, Manson H, Rehm J, Lin E, Kurdyak P. The burden of mental illness and addiction in Ontario. Revue canadienne de psychiatrie. 2013; 58(9):529-37. doi: 10.1002/ wps.20321.

16. Walker ER, McGee RE, Druss BG. Mortality in mental disorders and global disease burden implications: a systematic review and meta-analysis. JAMA Psychiatry. 2015;72(4):334-41. doi:10.1001/jamapsychiatry.2014.2502.

17. Jia H, Zack MM, Thompson WW, Crosby AE, Gottesman II. Impact of depression on quality-adjusted life expectancy (QALE) directly as well as indirectly through suicide. Soc Psychiatry Psychiatr Epidemiol. 2015; 50(6):939-949. doi:10.1007/s00127-015 $-1019-0$.
18. Chiao C, Lee $\mathrm{S}$, Liao $\mathrm{W}$ et collab. Social participation and life expectancy-the case of older adults in Taiwan from 1996 to 2003. Int J Gerontol. 2013;7(2):97-101. doi: 10.1016/j.ijge.2012.07.001.

19. Reynolds SL, Haley WE, Kozlenko N. The impact of depressive symptoms and chronic diseases on active life expectancy in older Americans. Am J Geriatr Psychiatry. 2008;16(5): 425-432. doi: 10.1097/jgp.0b013e31816ff32e.

20. Pérès K, Jagger $C$, Matthews FE. Impact of late-life self-reported emotional problems on Disability-Free Life Expectancy: results from the MRC Cognitive Function and Ageing Study. Int J Geriatr Psych. 2008;23(6):643-649. doi: 10.1002/gps.1955.

21. Steensma C, Loukine L, Orpana H, Lo E, Choi B, Waters C et collab. Comparing life expectancy and healthadjusted life expectancy by body mass index category in adult Canadians: a descriptive study. Popul Health Metr. 2013;11(1):21. doi: 10.1186/1478-7954 $-11-21$.

22. Tambay JL, Catlin G. Plan d'échantillonnage de l'Enquête nationale sur la santé de la population. Rapports sur la santé. 1995;7(1):31-43.

23. Béland Y. Enquête sur la santé dans les collectivités canadiennes : aperçu de la méthodologie. Rapports sur la santé. 2002;13(3):9-16.

24. Dai S, Robitaille C, Bancej C, Loukine L, Waters C, Baclic O. Note de synthèse : Rapport du Système national de surveillance des maladies chroniques : l'hypertension au Canada, 2010. Maladies chroniques au Canada. 2010; 31(1):54-55.

25. Kessler RC, Barker PR, Colpe LJ et collab. Screening for serious mental illness in the general population. Arch Gen Psychiatry. 2003;60(2):184-189.

26. Feeny D, Furlong W, Torrance GW et collab. Multiattribute and single-attribute utility functions for the Health Utilities Index Mark 3 system. Med Care. 2002;40(2):113-128.
27. Horsman J, Furlong W, Feeny D, Torrance G. The Health Utilities Index (HUI): concepts, measurement properties and applications. Health Qual Life Outcomes. 2003;1:54.

28. Feeny D, Huguet N, McFarland BH, Kaplan MS. The construct validity of the Health Utilities Index Mark 3 in assessing mental health in population health surveys. Qual Life Res. 2009; 18(4):519-526.

29. Mihalopoulos C, Chen G, Iezzi A, Khan MA, Richardson J. Assessing outcomes for cost-utility analysis in depression: comparison of five multiattribute utility instruments with two depression-specific outcome measures. Br J Psychiatry. 2014;205(5): 390-397. doi: 10.1192/bjp.bp.113 .136036

30. Joormann J, Quinn ME. Cognitive processes and emotion regulation in depression. Depress Anxiety. 2014; 31(4):308-315. doi: 10.1002/da.22264.

31. Simpson KR, Meadows GN, Frances AJ, Patten SB. Is mental health in the Canadian population changing over time? Revue canadienne de psychiatrie. 2012;57(5):324-331.

32. Chiang CL. The life table and its applications. Malabar (FL) : Krieger; 1984.

33. Hsieh JJ. A general theory of life table construction and a precise abridged life table method. Biom J. 1991; 33(2):143-162.

34. Sullivan DF. A single index of mortality and morbidity. HSMHA Health Rep. 1971;86(4):347-54.

35. Arriaga EE. Measuring and explaining the change in life expectancies. Demography. 1984;21(1):83-96.

36. Nusselder WJ, Looman CW. Decomposition of differences in health expectancy by cause. Demography. 2004;41(2):315-334.

37. Feng Y, Bernier J, McIntosh C, Orpana H. Validation des catégories d'incapacité dérivées des scores du Health Utilities Index Mark 3. Rapports sur la santé. 2009; 20(2):45-53. 
38. Comité directeur sur l'espérance de vie ajustée en fonction de l'état de santé de l'Agence de la santé publique du Canada. L'espérance de vie ajustée en fonction de l'état de santé au Canada : rapport de 2012 présenté par l'Agence de la santé publique du Canada. Ottawa (Ont.) : Agence de la santé publique du Canada; 2012. Consultable à la page : http:// canadiensensante.gc.ca/publications /science-research-sciences-recherches /health-adjusted-life-expectancy -canada-2012-esperance-vie-ajustee -fonction-etat-sante/index-fra.php

39. Goesling J, Clauw DJ, Hassett AL. Pain and depression: an integrative review of neurobiological and psychological factors. Curr Psychiatry Rep. 2013;15(12):1-8. doi: 10.1007/s11920 $-013-0421-0$.

40. Trivedi MH, Greer TL. Cognitive dysfunction in unipolar depression: implications for treatment. J Affect Disord. 2014;152:19-27. doi: 10.1016/j.jad.2013 .09 .012 .
41. Shah AJ, Ghasemzadeh N, ZaragozaMacias E, Patel R, Eapen DJ, Neeland $\mathrm{IJ}$, et collab. Sex and age differences in the association of depression with obstructive coronary artery disease and adverse cardiovascular events. J Am Heart Assoc. 2014;3(3):e000741. doi : 10.1161/JAHA.113.000741.

42. Patten SB, Brandon-Christie J, Devji J, Sedmak B. Évaluation pratique de la forme abrégée pour la dépression majeure du Composite International Diagnostic Interview auprès d'un échantillon de sujets choisis dans la collectivité. Maladies chroniques au Canada. 2000;21(2):73-77.

\title{
Demande de soumissions
}

\section{Promotion de la santé et prévention des maladies chroniques au Canada}

\author{
Les rédacteurs de Promotion de la santé et prévention des \\ maladies chroniques au Canada sont heureux d'annoncer que \\ la revue invite à nouveau la soumission d'articles de tous les \\ auteurs, indépendamment de leur rattachement.
}

Pour plus d'information et pour soumettre un article, veuillez consulter :

phac-aspc.gc.ca/publicat/hpcdp-pspmc/autinfo-fra.php\#subman

Recherche, politiques et pratiques 